\title{
Structural Performance Characteristics of Nanomaterials and Its Application in Traditional Architectural Cultural Design and Landscape Planning
}

\author{
Chaohai Ou ${ }^{1,2}$ and DeWei Wang $\mathbb{D}^{3}$ \\ ${ }^{1}$ School of Humanities and Arts, Chongqing University of Science \& Technology, Chongqing 401331, China \\ ${ }^{2}$ School of Architectural and Urban Planning, Chongqing University, Chongqing 400044, China \\ ${ }^{3}$ School of Civil Engineering and Architecture, Chongqing University of Science and Technology, Chongqing 400044, China
}

Correspondence should be addressed to DeWei Wang; 2008074@cqust.edu.cn

Received 19 January 2021; Revised 11 March 2021; Accepted 24 March 2021; Published 8 April 2021

Academic Editor: Sang-Bing Tsai

Copyright (C) 2021 Chaohai Ou and DeWei Wang. This is an open access article distributed under the Creative Commons Attribution License, which permits unrestricted use, distribution, and reproduction in any medium, provided the original work is properly cited.

\begin{abstract}
In recent years, with the rapid development of industrial technology, industrial materials have also begun to develop in more and more refined directions. Nanotechnology is one of the most developed core technologies in industrial technology, and these characteristics of nanomaterials make them shine in many fields, especially in the field of construction. Nowadays, conventional materials still have the problems of heavy weight and high thermal conductivity as thermal insulation materials for external walls. At the same time, it has outstanding performance that conventional materials do not have. Nanomaterials are widely used in various fields with rapid growth due to their many excellent properties and have produced huge economic benefits and social significance. This article describes the basic characteristics of nanomaterials, including surface effects, quantum size effects, small size effects, and macroscopic quantum tunneling effects. As nanomaterials and nanotechnology become more and more mature, the research on architectural coatings in our country has been slightly strengthened. Nanomaterials have a huge impact on future economic development and have become the most promising objects in the current material field. The experimental results prove that the foaming ability of nanomaterials is much better than that of ordinary materials, and the fault tolerance rate of the cement paste is also the highest. This can also positively show that the relationship between nanotechnology and construction engineering is getting closer and closer, and the combination of nanomaterials and construction materials shows people the broad prospects of construction materials for the development of ecological construction materials that improve the living environment and improve the quality of life.
\end{abstract}

\section{Introduction}

The so-called nanocrystals refer to particles that can be observed with an electron microscope or whose diameter is between 1 and $100 \mathrm{~nm}[1]$. Nanocrystals and the tendons, skin, bones, and proteins of animals we are familiar with are widely found in nature. Since 1861, humans have been studying so-called colloids (nanosystems) in the field of chemistry but only after the 1960s, did they begin to consciously prepare and study nanocrystals. With the deepening of research on nanotechnology [2], there is more and more knowledge about nanomaterials, and more nanomaterials have been studied, which are applied in many fields. Nanomaterials are applied to the coating industry to develop functions. Better performance coatings provide a new approach [3]. Scientific and technical communities in different countries attach great importance to the study of nanomaterials. The discovery of nanometers not only opened up more opportunities for materials science and high-tech applications but also opens up a complex question for physical physics.

The development of nanomaterials industry abroad has a long history, which is mainly used in the field of building energy-saving applications [4]. European wall insulation 
materials are mainly polystyrene and rock wool. This type of slurry insulation has low thermal conductivity, good strength, and safety factor and has excellent performance. For example, polyurethane slurry insulation material of polyurethane foam does not have much braking effect and has good flame retardancy and resistance [5]. Calcium silicate has extremely high waterproof performance. Takasu proposed that the number of free electrons in ultrafine metal particles is too small, and it is difficult to take out or inject electrons from particles smaller than $10 \mathrm{~nm}$. This effect is the famous Kubo effect [6] compared to the influence of heat and superconductivity. Nicolopoulos et al. use the method of evaporation and condensation in an inert gas to obtain relatively pure ultrafine particles, and through the electron diffraction technique, the crystal structure of the crystal particles is measured [7]. When Peets studied the superconductivity of $\mathrm{Al}$, he found that the critical superconducting temperature of nano-Al is 2.7 times that of crystalline Al. The critical superconducting temperature of nano-Al is 2.7 times than that of crystalline $\mathrm{Al}$ [8].

The vigorous development of nanomaterials in our country has produced many different types of insulation materials that can be used in building energy conservation, and a large-scale insulation material production and technical system has been initially formed. The current inorganic external wall thermal insulation materials mainly include traditional wall thermal insulation materials and new thermal insulation wall materials. At the same time, in order to enhance their thermal insulation performance, an infrared sunscreen with the best particle size distribution is added to the material, through its refraction, and absorption is used to disperse the infrared radiation that has the effect of heat so as to minimize the amount of radiation, in order to optimize the effect. An et al. summarized various methods of producing ultrafine particles, improved the technology of preparing traditional nanoparticles by direct evaporation from resistance cables, and proposed a method "the general technology" for producing ultrafine particles by evaporation from an inert gas atmosphere under reduced pressure [9]. Chen et al. adopts the pressure forming method of inert gas condensation system to prepare nanomaterials with specific properties and, for the first time, proposed the concept of nanosolid, which opened a historic page for the research of nanomaterials [10]. Ma et al. applied nanomaterials in the construction process of oilfield cementing and found that its effect is significant, which has promoted the considerable progress of nanotechnology [11].

With the deepening of research on nanotechnology, there is more and more knowledge about nanomaterials, and more nanomaterials have been studied and applied in many fields. The foaming ratios of nanomaterials AOS and K12 are relatively high, 5.9 and 5.4, respectively, while the foaming ratio of ordinary materials is the smallest (2.2), and AES is 4.9 between the two, which proves that AOS has the strongest foaming ability and the fault tolerance rate of cement paste is correspondingly the highest. It further illustrates that nanomaterials have a broad market for building materials. Nanotechnology will not only bring more and more new building materials but also improve people's living environment. Nanomaterials will definitely become the key development direction of current building materials.

\section{Structural Performance Characteristics of Nanomaterials and Their Application in Traditional Architectural Cultural Design and Landscape Planning}

2.1. Structural Performance Characteristics of Nanomaterials. The structure of nanomaterials is different from conventional materials and belongs to the transitional area of materials from the macroworld to the small world. Therefore, many traditional physical and chemical theories are no longer applicable to this nonmacro- and nonmicrofield $[12,13]$. At the same time, the factors affecting the nanocrystalline structure are very complex. The type of substance, particle size, distribution of nanocrystals, preparation methods, and various analysis methods will affect all analysis results.

2.1.1. Classification of Nanomaterials. Nanomaterials can be divided into two types of structural forms: the first type of nanomaterial structure consists of two types of structures composed of particles and grain boundaries, and the size of all structural elements is nanometers [14]. The situation where the percentage of the total atomic volume is only $1 \%$ is completely different. Assuming that the crystal grains are spherical or cubic, the atomic volume fraction $C$ of the inner interface of the nanocrystallite is

$$
C=\frac{3 \Delta}{d}
$$

In the formula, $C$ represents the atomic volume fraction of the inner interface, $\Delta$ represents the average grain boundary thickness, and $d$ represents the average grain diameter. After the $\Delta$ value is appropriately corrected, when the crystal grain diameter $d=5 \mathrm{~nm}, C=50 \%$. When $d=10 \mathrm{~nm}, C=30 \%$. It can be seen that the smaller the average grain diameter, the more the grain boundaries, and the more the atoms on the crystal interface [15]. This highconcentration interface structure makes the material have a dense structure, and the properties of the material have undergone great changes. The X-ray diffraction of this type of material shows the phenomenon of diffraction peak expansion caused by the small size.

The second nanomaterial is a random low-density network structure material with a large number of nano-sized cavities. The entire structure is composed of nanocrystalline particles and nanosystems, sometimes including nanoskeletal structures and transfer atomic clusters smaller than nanocrystalline particles. It has a low density, a huge random network structure, and a complicated channel and resource structure [16, 17]. The concentration of surface structure in the material is very high from $20 \%$ to $50 \%$. At the same time, it also has a large number of interface structures, and the high concentration of surface structure atoms significantly changes the properties of the material [18]. 


\subsubsection{Structural Characteristics of Nanomaterials}

(1) Small Size Effect. The macroscopic size of the nanocrystalline unit constituting the nanosolid is in the nanometer range. The particle size is equal to or smaller than the light wavelength, the de Broglie wavelength, and the physical characteristics of the coherence or transmission depth of the superconducting state. Its cyclic state will be destroyed, the almost continuous energy levels will be divided into individual energy levels, and the kubo effect will be produced $[19,20]$. This is the extreme case of the small size effect. The density of atoms near the surface particle layer of amorphous nanoparticles is reduced, which has little effect on physical properties (such as sound, light, electromagnetics, and thermodynamics); for example, changes in plasmonic resonance frequency will significantly increase light absorption and produce absorption peaks; abnormal conductivity and diffusion; transition from superconducting phase to normal phase; and changes in the sound spectrum.

(2) Macroscopic Quantum Tunneling Effect. The ability of microscopic particles to penetrate the barrier is called the tunneling effect. Some macroscopic quantities, such as the magnetization of microparticles and the magnetic flux in quantum coherent devices, have a tunneling effect. This is of great significance to basic research and practicality [21, 22]; for example, it limits the time limit for tapes and disks for information storage, and it establishes the limit for the further miniaturization of existing microelectronic devices. When manufacturing semiconductor integrated circuits, when the size of the circuit is close to the wavelength of electrons, electrons will pass through the tunnel effect. Overflowing the device makes the device inoperable.

(3) Surface Effect. Surface effect refers to the phenomenon that the ratio of surface atom number to total atom number of nanoparticles increases sharply with the decrease in particle size. Due to the increase in the number of surface atoms, high surface energy, and incomplete atomic coordination number, there is a serious vacancy state, very unstable, extremely active, and easy to combine with other atoms [23], resulting in some novel effects.

\subsection{Application of Nanotechnology in Construction}

2.2.1. Modification of Paint. In the production of coatings, nanometer coatings can be made by adding nanoscale anatase $\mathrm{TiO}_{2}$. Studies have proved that nano-anatase $\mathrm{TiO}_{2}$ has good chemical stability and strong photocatalytic ability.

Due to the quantum size effect, when a photon with energy of magnitude $h_{\gamma}$ or a photon with energy $E_{E}$ exceeding the semiconductor band gap is shot into $\mathrm{TiO}_{2}$, the electron is excited from the $\mathrm{VB}$ valence band to the $\mathrm{CB}$ conduction band, leaving a hole behind. The holes in the valence band are strong oxidants (from +1.0 to $+1.3 \mathrm{~V}$; the relationship with $\mathrm{NHE}$ depends on the $\mathrm{pH}$ value of the semiconductor), while the electrons in the conduction band are good reducing agents (starting from +0.5 to $-1.5 \mathrm{~V}$, regarding NHE) $[24,25]$. The transition of the valence band of $\mathrm{TiO}_{2}$, the strong redox agent produced, causes the water and oxygen in the air to react to generate active oxygen and active $\mathrm{OH}^{-}$groups:

$$
\begin{aligned}
& \mathrm{O}_{2}+\mathrm{e}^{-} \longrightarrow \mathrm{O}_{2}^{-} \\
& \mathrm{H}_{2} \mathrm{O}+\mathrm{P}^{+} \longrightarrow \mathrm{OH}^{-}+\mathrm{H}^{+}
\end{aligned}
$$

The extremely strong redox properties of active oxygen and active $\mathrm{OH}^{-}$groups can destroy the C-C, C-H, C-N, C-O, and $\mathrm{O}-\mathrm{H}$ bonds of organic substances. Therefore, the use of sunlight can even completely mineralize organic compounds in many cases. Sewage treatment and other environmental protection projects are of great significance. Adding anatase $\mathrm{TiO}_{2}$ in the paint production, this type of paint can effectively decompose organic matter, kill bacteria and viruses, and eliminate air pollutants, such as halides, sulfides, aldehydes, polycyclic aromatic hydrocarbons, and ammonia $[26,27]$. In addition, adding nano- $\mathrm{SiO}_{2}$ to the coating can improve the antiaging performance, and the drying method, the smoothness and durability of the coating, and the wear resistance can be increased by more than 10 times.

2.2.2. Modification of Ceramics. The anatase-type nano$\mathrm{TiO}_{2}$ photocatalyst can be coated and cast on the surface of ceramics by glazing or spraying methods to make a ceramic wall that can kill bacteria and viruses and decompose organic volatiles and other harmful gases in the air. Sanitary ware is a very good building material, which can be used in public places, swimming pools, toilets, and rooms. However, since the photocatalyst needs the irradiation of ultraviolet light to function, the sterilization and purification effect is not good in the dark place [28]. The sterilization and air purification performance of this ceramic material can be improved by simultaneously adding silver, copper, or other antibacterial agents irradiating with ultraviolet light. Because nanoparticles will grow up at high temperatures, in order to ensure the excellent properties of nanomaterials and effectively fix nanoparticles, heat treatment is often used, and the temperature should generally exceed $450^{\circ} \mathrm{C}$.

$\mathrm{Al}_{2} \mathrm{O}_{3}$ is a ceramic material with a wide range of uses and low prices. In order to improve its performance, humans use small $\mathrm{Al}_{2} \mathrm{O}_{3}$ and nanoscale $\mathrm{SiC}$ to synthesize in the laboratory, then heat press at $180^{\circ} \mathrm{C}$, and then anneal in an argon atmosphere at $1300^{\circ} \mathrm{C}$ for 2 hours. The fracture strength is increased to $1540 \mathrm{MPa}$ (and the fracture strength is only $560 \mathrm{MPa}$ ), which improves its brittleness and can even become ceramic springs, knives, etc., so that ceramic materials have new mechanical properties, called "indestructible ceramics." Although this technology is currently difficult to achieve industrial production, it shows the delightful world of modified ceramics.

2.2.3. Modification of Glass. Using sol-gel technology or other coating technology, a transparent coating containing anatasetype nano- $\mathrm{TiO}_{2}$ crystals can be formed on the glass. This kind of glass is characterized by photocatalytic reaction under the irradiation of ultraviolet light, which produces active oxygen and active $\mathrm{OH}^{-}$groups, which can decompose the oil and other 
organic matter attached to the glass surface, reduce the waterrepellent angle of the glass surface, and make the glass have the self-cleaning and superhydrophilic effects greatly reduce the workload of cleaning and cleaning [29]. It can be used for glass curtain wall, road lighting lampshade, automobile glass and reflector, solar battery, and solar water heater.

\subsubsection{Building Materials Capable of Purifying Nitrogen} Oxides. In recent years, the deterioration of air quality has become a focus of attention. Among them, nitrogen oxide $(\mathrm{NOx})$ is an important indicator, which is very harmful to human health. Utilizing the anatase-type nano- $\mathrm{TiO}_{2}$ photocatalyst ability not only can efficiently decompose organic matter and kill bacteria but also can effectively oxidize stable and difficult to remove $\mathrm{NOx}$ into water-soluble $\mathrm{NO}_{3}$, which can be easily removed with water to eliminate pollution, the role of air purification [30]. Using different building materials such as nano- $\mathrm{TiO}_{2}$ and photocatalyst carriers can be made into various ecological building materials that can purify NOx.

\subsection{Application of Cellulose in Construction}

2.3.1. Aggregate Structure of Cellulose. The aggregated structure of cellulose refers to the cellulose supramolecular structure composed of the relative arrangement of cellulose molecules in space. Mainly study how cellulose molecules are arranged to form the structure of crystalline and amorphous regions. The cellulose molecules in the crystalline area are arranged neatly and regularly in space, with smaller molecular spacing and higher density, which can reach $1.59 \mathrm{~g} / \mathrm{cm}^{3}$. Through X-ray diffraction research and analysis, clear diffraction peaks can be seen. In addition, because the cellulose molecular chain contains a large number of hydroxyl groups, strong intramolecular and intermolecular hydrogen bonds can be formed in the crystalline region so that the cellulose molecules form a dense and highly regular structure [31, 32]. In the amorphous zone, also known as the amorphous zone, the cellulose molecules in this part are not neatly arranged, looser, have a larger distance between the molecules, and have a lower density, generally $1.50 \mathrm{~g} / \mathrm{cm}^{3}$. The intermolecular hydrogen bonds are weak so that part of the hydroxyl groups can form hydrogen bonds with other molecules, showing strong hydrophilicity.

2.3.2. Cellulose Derivatives. Because cellulose has good physical and chemical stability and cannot be melted and insoluble in common reagents, cellulose is usually converted into cellulose derivatives for use in industrial applications [33]. Although traditional natural cellulose is still one of the main raw materials of the chemical textile industry, the raw materials of man-made cellulose derivatives have become the main development direction of chemical fibers.

(1) Cellulose Acetate. It has a wide range of uses in coatings, construction industry, and other fields. Because the hydroxyl group on the glucose ring of the cellulose molecular chain is replaced by the acetyl group, the number of hydroxyl groups in the molecule is reduced, and the intermolecular and intramolecular hydrogen bonding is greatly weakened. At the same time, the appearance of acetyl group increases the intermolecular distance, which greatly improves the solubility and processability of CAT.

(2) Cellulose Acetate Nanofiber. People gradually shifted their focus to the research of nanomaterials. Generally speaking, when a substance reaches the nanometer scale, its properties will often undergo mutations, and special properties will appear, which are different from the original atoms and molecules and also different from macroscopic substances. Materials with such special properties are called nanomaterials. Nanostructured materials can exhibit some unique properties, such as surface effects and small size effects. It has many unique properties that traditional materials do not have, making it the most dynamic research topic in the field of new materials research today, has a very important impact on future economic and social development, and has produced important applications in many fields.

\subsection{Nanomaterials}

2.4.1. Characteristics of Nanomaterials. Nanomaterials are usually very small in size and larger on specific surfaces and have a higher surface energy. The number of superficial people accounted for a large percentage of the total. When the particle size is less than $10 \mathrm{~nm}$, the percentage of surface atoms increases sharply, and these surface atoms are in a very empty state. They are extremely active and very unstable [34]. Once you meet other people and ally with them to stabilize, this effect is called a surface effect.

In nanomaterials, when the particle size reaches a certain physical characteristic, the energy levels of electrons adjacent to the Fermi metal plane change from an almost continuous state to a discrete state, and nanoparticles have discontinuous molecules with a higher efficiency. The orbital and low molecular orbital are asymmetric, and the energy gap is the quantum size phenomenon of nanomaterials. When the ultrafine particle size is equal to or smaller than the physical size such as the wavelength of the light wavelength, the periodic boundary crystal condition will be destroyed, and the atomic density near the surface layer of the amorphous nanoparticle will decrease. It makes sound, light, electricity, magnetism, thermodynamics, and other physical properties show little influence.

2.4.2. Application of Nanomaterials. Looking at the literature and books on nanoelectrocatalysts at the forefront of science, the most mentioned word is energy. Energy is no longer a problem of a certain country but has become a problem of global concern. When a crisis occurs, there is no country, which can take care of itself. The vast number of scientific researchers are working against clean energy. As a very important role in clean energy, nano-electrocatalysts are attracting widespread attention. No matter whether the heat increases or decreases, 
nano-electrocatalysts will become the solution. The key to the energy issue is nanomaterials and they have attracted great attention since their birth [35]. With the continuous advancement of science and technology, the research on nanoelectrocatalysis by scientific researchers has become more and more in-depth, mainly used in the following aspects: hydrogen and oxygen evolution electrocatalyst, electrocatalytic carbon dioxide reduction catalyst, and microbial fuel cell electrocatalyst.

\section{Application of Nanomaterials in Traditional Architectural Cultural Design and Landscape Planning}

3.1. Test Subject. This experiment selects nanomaterials AOS, AES, and k12 and the foamability of the three nanomaterials has an irreversible effect on the cement slurry, mainly considering the stability of the foam produced by foaming and its fault tolerance with the cement slurry, to ensure that the life of the foam in the cement is not less than the hardening and hardening time of the cement, and it will not negatively affect the performance of the cement. The foam ratio is the ratio of the total volume of foam to the volume of the original foam aqueous solution. This ratio is used as a quantitative indicator of the foaming ability of the foaming agent. If the ratio is higher, the foaming and foaming ability of the foaming agent is stronger. The total volume of the liquid film discharged between bubbles is the volume of bubble secretion. As time goes by, the smaller the change in foam secretion, the better the foam stability.

3.2. Experimental Method. Weigh $1 \mathrm{~g}$ of each of the three nanomaterials, add water and stir foam at a ratio of $1: 100$, stir in a stirring vessel at a constant speed for 5 minutes to foam, and pour it into a $500 \mathrm{ml}$ graduated cylinder immediately after stirring. Record the initial foam volume and the remaining liquid volume at the bottom and then read and record the data again at 5 minutes, 20 minutes, and 40 minutes. The volume of the foam volume reduced after 20 minutes and 40 minutes in a natural environment is used to represent the foam stability.

3.3. Gather Data. The statistical data used in this article have a different unit dimension for each index data. After calculating the data in the previous steps, we can get the similarity between users. Select several users closest to user $u_{a}$ interests and preferences to form set $N_{a}$. Then, calculate the score of user $N_{a}$ on $j$ according to the score of the user in the set $u_{a}$ on the unrated item $j$, and the prediction formula is shown as

$$
p_{a, i}=\frac{\sum_{b \in N_{a}} \operatorname{sim}_{u_{a}, u_{b}} r_{b, i}}{\sum_{b \in N a}\left|\operatorname{sim}_{u_{a}, u_{b}}\right|}
$$

where $p_{a, i}$ is the predicted score of user $a$ for the unrated $i$ item. In the recommendation system, users scoring preferences are sometimes different; for example, some users are accustomed to give higher ratings to items, while some are accustomed to give lower ratings. In order to reduce the difference between users scoring preferences and improve the accuracy of scoring predictions, the method of formula (4) introduces the users average rating $\bar{r}$, and the specific form is shown as

$$
p_{a, i}=\overline{r_{a}}+\frac{\sum_{b \in N_{a}} \operatorname{sim}_{u_{a}, u_{b}}\left(r_{b, i}-\overline{r_{b}}\right)}{\sum_{b \in N_{a}}\left|\operatorname{sim}_{u_{a}, u_{b}}\right|} .
$$

3.4. Statistical Method. SPSS23.0 software was used for data processing, and the count data were expressed as a percentage (\%), $k$ is the number of data in this experiment, $\sigma^{2}$ is the variance of all survey results, and $P<0.05$ indicates that the difference is statistically significant. The calculation formula of reliability is shown as

$$
a=\frac{k}{k-1}\left(1-\frac{\sum \sigma_{i}^{2}}{\sigma^{2}}\right) \text {. }
$$

\section{Application of Nanomaterials in Traditional Architectural Culture Design and Landscape Planning}

4.1. Evaluation Index System Based on Index Reliability Testing. Reliability refers to the stability and reliability of the questionnaire. This article adopts the $\alpha$ coefficient method created by Cronbach. The $\alpha$ coefficient can be obtained by reliability analysis in SPSS software. It is generally believed that the $\alpha$ coefficient above 0.8 indicates that the effect of index setting is very good, and above 0.7 is also acceptable. The results are shown in Table 1.

It can be seen from Table 1 that the index effects set for the three nanomaterials in this experiment are acceptable $(\alpha>0.7)$, which proves that some environmental factors and the effects of different stirring speeds during the experiment are in the acceptable range. Within this, the prerequisites for the experiment are met, which provides a basis for subsequent experimental analysis.

4.2. Performance Test Results of Different Nanomaterials. Here, we test and analyze the performance of nanomaterials, by reading and recording the data again at 5 minutes, 20 minutes, and 40 minutes, and the volume of the foam after 20 minutes and 40 minutes in the natural environment is used to represent the foaming stability. We compare and analyze the volume of foam produced, the amount of remaining liquid, and the expansion ratio, and the results are shown in Figure 1.

It can be seen from Figure 1 that the foaming ratios of nanomaterials AOS and K12 are relatively high, 5.9 and 5.4, respectively, while the foaming ratio of ordinary materials is the smallest (2.2), and the AES is 4.9 between the two, indicating that AOS has the highest foaming ability. The fault tolerance rate of cement paste is also the highest. Ordinary materials have the strongest foaming ability and correspondingly the highest fault tolerance of cement paste. Based on these nanomaterials, AOS has the best foaming 
TABLE 1: Summary table of reliability test results.

\begin{tabular}{lcc}
\hline Group & Index combination & Alpha coefficient $(\alpha)$ \\
\hline \multirow{2}{*}{ AOS } & Foam volume & 0.8447 \\
& Remaining liquid & Foaming multiple \\
\hline \multirow{2}{*}{ AES } & Fault tolerance & 0.7644 \\
& Foam volume & \\
\hline & Remaining liquid & 0.7224 \\
k12 & Foaming multiple & Fault tolerance \\
& Foam volume & Remaining liquid \\
\hline
\end{tabular}

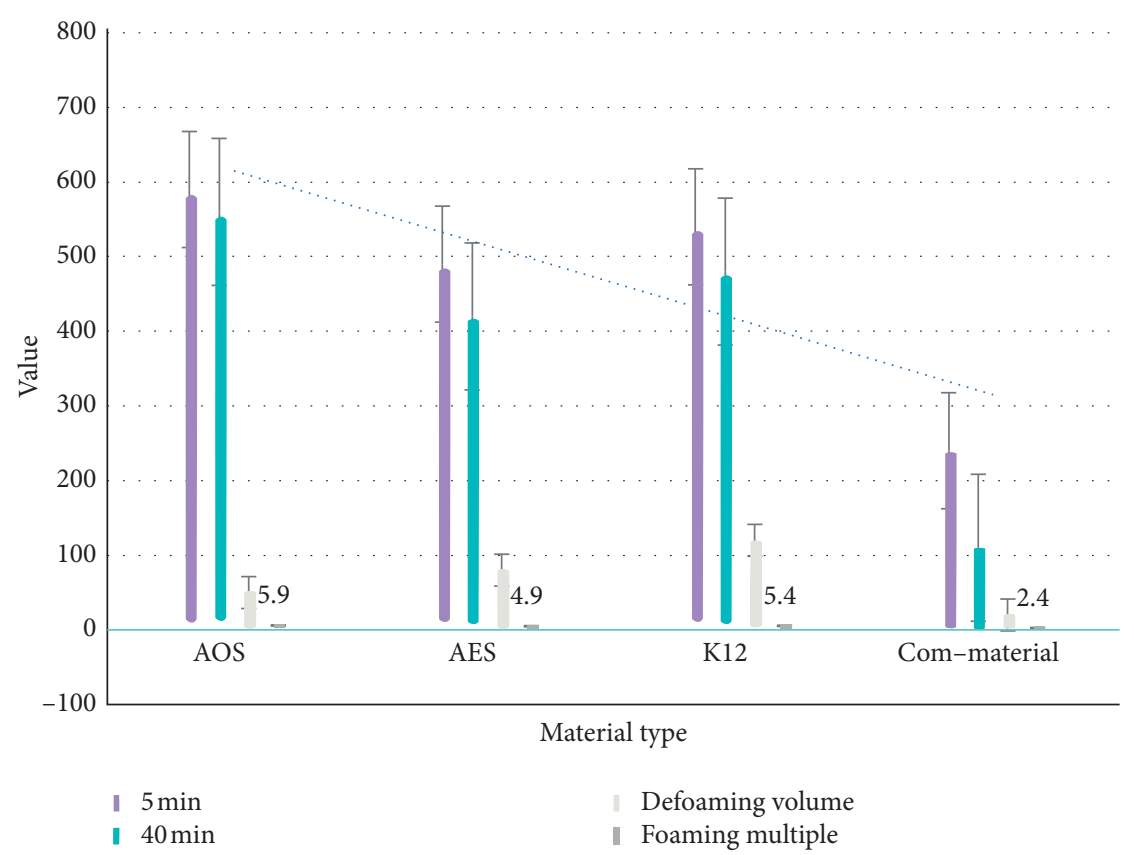

Figure 1: Analysis diagram of performance test results of different nanomaterials.

performance and foam stabilization performance, and it should be selected as the nanomaterial for subsequent experiments.

\subsection{Effect of Physical Foaming Process on the Properties of Nanomaterials}

\subsubsection{Results of the Amount of Nanomaterial AOS Parameter} Powder. By adopting single-factor and multifactor control variable method and while keeping the water-cement ratio and cement quality unchanged, the performance test of the sample prepared with the content of nanomaterial AOS powder as the independent variable was carried out. The relationship between apparent density, porosity, compressive strength, volumetric water absorption, and thermal conductivity is shown in Figure 2.

It can be seen from Figure 2 that, with the gradual increase in the amount of nanomaterial AOS powder, the apparent density, compressive strength, volumetric water absorption, and thermal conductivity all show a downward trend, while the porosity gradually increases. At the same time, the volume of foam introduced by nanomaterials increases, and the proportion of closed bubbles that are stable in the slurry increases. Therefore, the volume of gas contained in the unit slurry increases, and the porosity in the hardened cement substrate continues to increase, resulting in a decrease in density.

\subsubsection{Results of Nanomaterial $k 12$ Parameter Powder} Quantity. By adopting single-factor and multifactor control variable methods and while keeping the water-cement ratio and cement quality unchanged, the performance test of the sample prepared with the content of nanomaterial $\mathrm{k} 12$ powder as the independent variable was carried out. The relationship between apparent density, porosity, compressive strength, volumetric water absorption, and thermal conductivity is shown in Figure 3. 


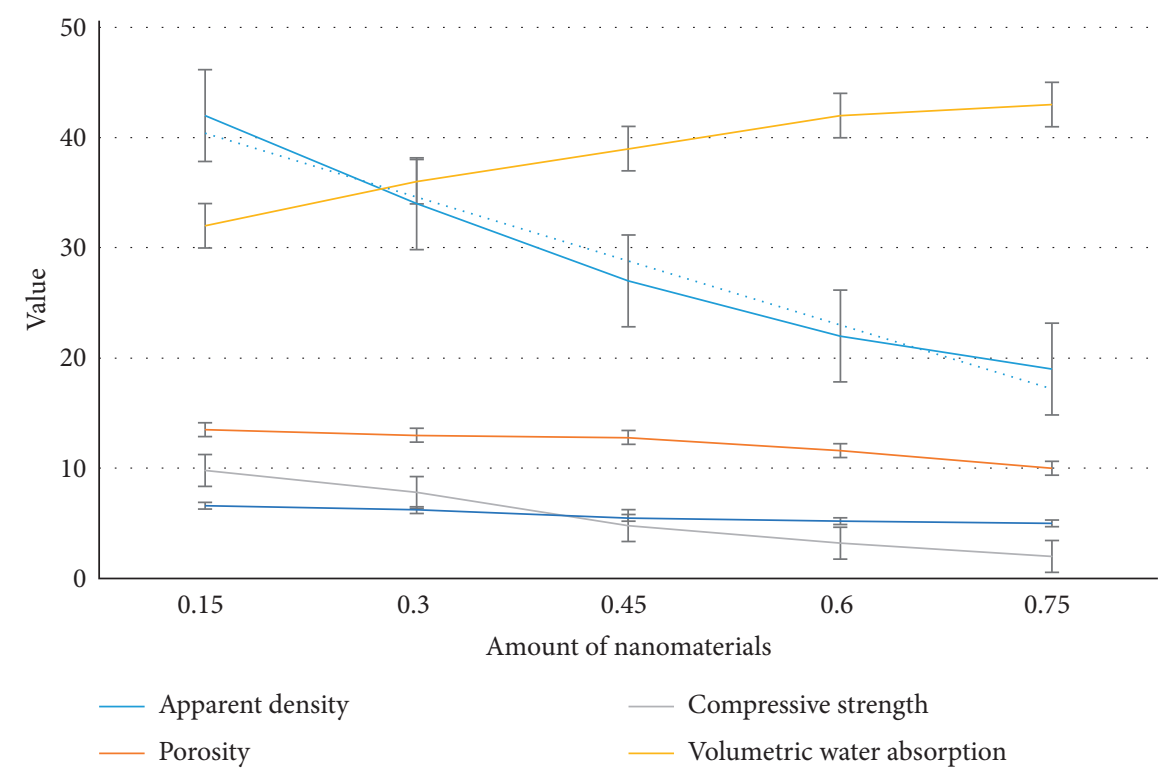

FIGURE 2: Result analysis diagram of AOS parameter powder amount of nanomaterials.

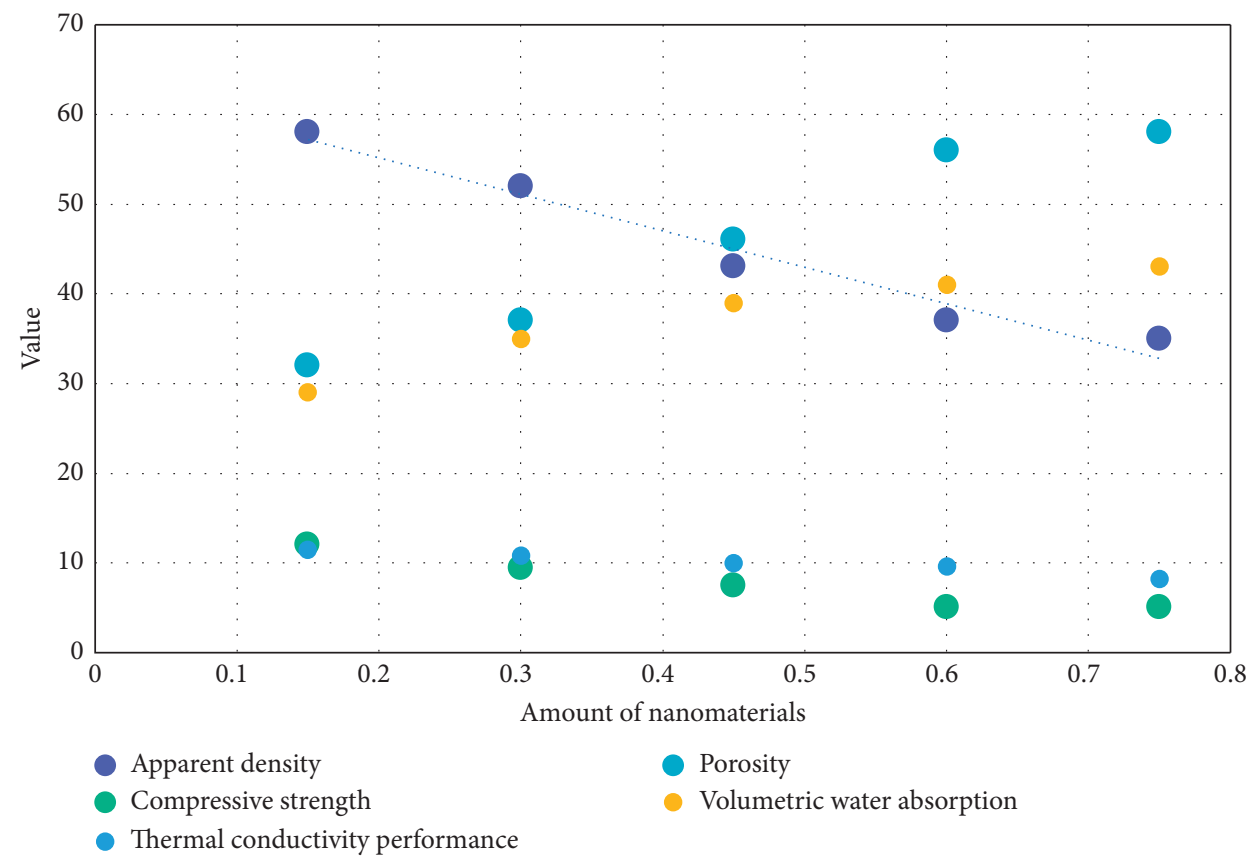

FIGURE 3: Result analysis diagram of nanometer material k12 parameter powder quantity.

It can be seen from Figure 3 that, with the gradual increase in the $\mathrm{k} 12$ content of nanomaterials, the apparent density, compressive strength, and thermal conductivity continue to decrease, while the porosity and volumetric water absorption continue to increase. The nanomaterial k12 will rapidly undergo chemical reactions and decompose in a large amount, and the volume of oxygen released will continue to increase so that the volume of gas contained in the unit volume of cement paste increases, and the porosity of the hardened cement substrate increases. The density will naturally continue to decrease.
4.3.3. Results of AES Parameters of Nanomaterials. By adopting single-factor and multifactor control variable method, while keeping the water-cement ratio and cement quality unchanged, the performance test of the sample prepared with the content of nanomaterial AES powder as the independent variable was carried out. The relationship between the apparent density, porosity, compressive strength, volumetric water absorption, and thermal conductivity is shown in Figure 4.

It can be seen from Figure 3 that, with the gradual increase in the content of AES, the apparent density, 


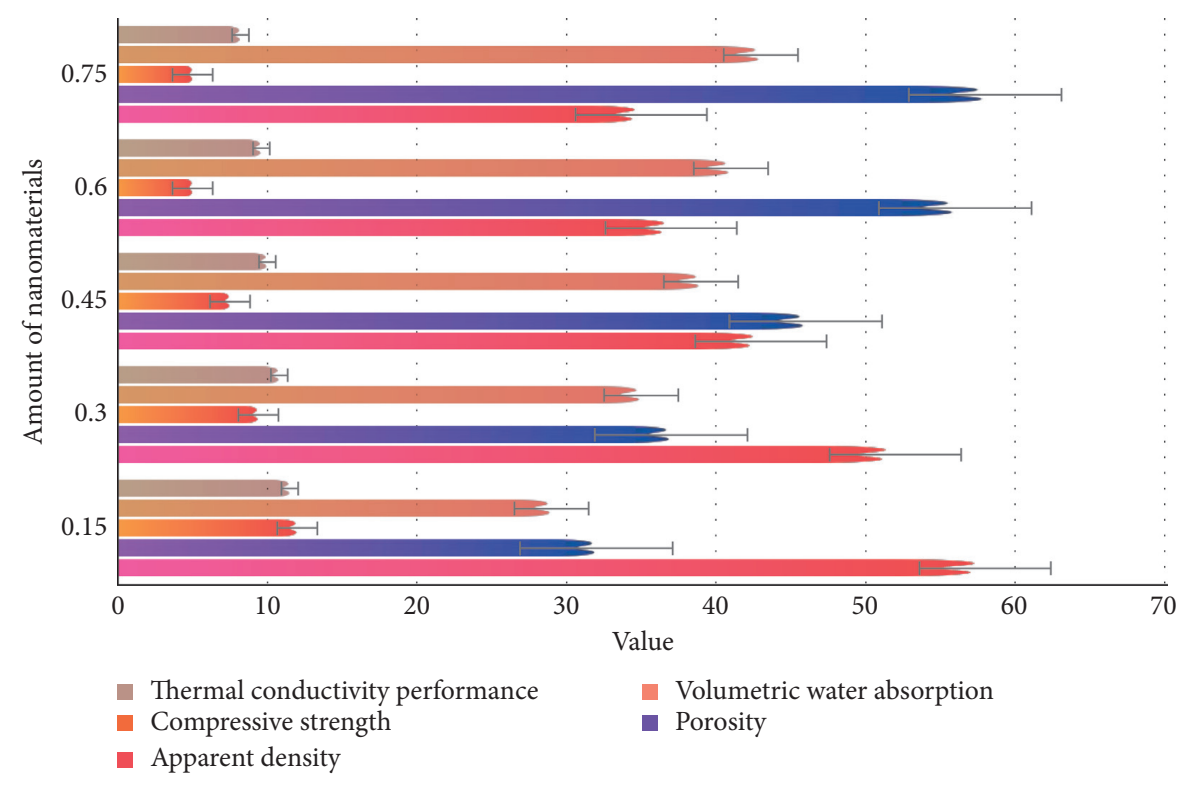

FIGURE 4: Result analysis diagram of AES parameters of nanomaterials.

compressive strength, and thermal conductivity continue to decrease, while the porosity and volumetric water absorption continue to increase. The reason for the above results is that, with the decrease in the chemical foaming agent and the increase in the physical foaming agent content, the consistency of the slurry increases, which has a certain restraint effect on the chemical gas expansion force. The number of macroporous interconnected cells is reduced, and the pore structure becomes more uniform and dense, resulting in an increase in the number of closed bubbles formed in the cement paste and an increase in the number of closed cells. At the same time, the communication rate between the cells is reduced, and the water absorption rate is greatly reduced.

\section{Conclusions}

The development of nanotechnology has opened a door to innovative ideas in art and design. Design is a bridge that connects public vision with nanotechnology. This article analyzes the aesthetic laws of nanostructures from the aspects of structure, aggregation, and fragmentation and discusses nanotechnology and environmental art from two perspectives: the mutual inspiration of research and design concepts, the mutual reference of technology and artistic language, and the use of exhibition halls. Take design practice as an example to discuss the application of nanotechnology in environmental design. Although the relevant research and exploration is very superficial, it is not difficult to see that the tiny world provides unlimited imagination space for environmental design, and the application of nanotechnology to environmental design has a broad prospect. If looking for inspiration from traditional culture is a monument and perception of history, it is a memory of history, and then looking for inspiration in the high-tech field is both a desire for the future and a desire for the unknown. Human life has gradually entered the nanoage, and this exploration and pursuit will affect our lives on a deeper level.

The unique structural features and special properties of nanomaterials have had a significant impact on the established theoretical systems of traditional physics, chemistry, and engineering. The new phenomena and new laws of nanomaterials are pushing people to establish new theories. "Who lost the nano. Who lost the future" has become the consensus of all countries in the world. As Academician Qian Xuesen predicted, "Nanotechnology will be another industrial revolution in the 21st century," which shows the importance of nanotechnology. Nanomaterials are the foundation of all nanotechnology and have been widely used in various fields. However, from the perspective of basic research and the practical application of nanomaterials, its research still faces many problems and severe challenges; for example, the synthesis method is complicated, the controllable preparation of monodisperse nanoparticles or nanotubes is not fully studied, the growth mechanism has not been fully understood, and there is lack of systematic performance research. However, we have reason to believe that, with the continuous advancement of science and technology and the continuous improvement of preparation and modification technologies, nanomaterials will be more widely used in more fields in the future.

Analyzing the mechanism of the difference in gas sensitivity of nanomaterials with different phase structures, it can be known that, for different gases, there are many factors that affect the selectivity, but for the three samples with different phase structures, there is no significant difference in the selectivity of ethanol. The reason for the increased response to various gases is that its symmetry is relatively low, the electric dipole moment is high, and the system is relatively active, resulting in an increase in the adsorption force between it and polar gas molecules. The sensitization reaction on the surface of the material is more thorough, 
which in turn shows a greater response to gas. Based on the increase in the specific surface area and the increase in surface oxygen defects, the gas sensitivity performance has been significantly improved and optimized.

\section{Data Availability}

The data underlying the results presented in the study are available within the manuscript.

\section{Conflicts of Interest}

The authors declare that they have no conflicts of interest.

\section{References}

[1] B. Gao, N. Xu, and P. Xing, "Shock wave induced nanocrystallization during the high current pulsed electron beam process and its effect on mechanical properties," Materials Letters, vol. 237, no. 15, pp. 180-184, 2019.

[2] S. Chen, M. K. Hassanzadeh-Aghdam, and R. Ansari, "An analytical model for elastic modulus calculation of $\mathrm{SiC}$ whisker-reinforced hybrid metal matrix nanocomposite containing SiC nanoparticles," Journal of Alloys and Compounds, vol. 767, pp. 632-641, 2018.

[3] J. Zhao, J. Huang, Y. Xiang et al., "Effect of a protective coating on the surface integrity of a microchannel produced by microultrasonic machining," Journal of Manufacturing Processes, vol. 61, pp. 280-295, 2021.

[4] C. Li, F. Sun, J. M. Cioffi, and L. Yang, "Energy efficient MIMO relay transmissions via joint power allocations," IEEE Transactions on Circuits and Systems II: Express Briefs, vol. 61, no. 7, pp. 531-535, 2014.

[5] N. Gao, B. Cheng, H. Hou, and R. Zhang, "Mesophase pitch based carbon foams as sound absorbers," Materials Letters, vol. 212, pp. 243-246, 2018.

[6] Y. Takasu, "Catalytic etching of various carbon materials by ultrafine metal particles," Tanso, vol. 2016, no. 274, pp. 145-154, 2016.

[7] S. Nicolopoulos, P. P. Das, A. G. Pérez et al., "Novel TEM microscopy and electron diffraction techniques to characterize cultural heritage materials: from ancient Greek artefacts to maya mural paintings," Scanning, vol. 2019, no. 6-7, 13 pages, Article ID 4870695, 2019.

[8] D. C. Peets, E. Cheng, T. Ying et al., "Type-I superconductivity in Al\$_6\$Re," Physical Review, vol. 99, no. 14, pp. 1-9, Article ID 144519, 2019.

[9] Y. An, Y. Sun, M. Zhang, B. Adhikari, and Z. Li, "Effect of ball milling time on physicochemical properties of Cordyceps militaris ultrafine particles," Journal of Food Process Engineering, vol. 42, no. 4, pp. 1-10, Article ID e13065, 2019.

[10] L. Chen, Y. S. Choy, T. G. Wang, and Y. K. Chiang, "Fault detection of wheel in wheel/rail system using kurtosis beamforming method," Structural Health Monitoring, vol. 19, no. 2, pp. 495-509, 2020.

[11] C. Ma, B. Zhao, G. Long, X. Sang, and Y. Xie, "Quantitative study on strength development of earth-based construction prepared by organic clay and high-efficiency soil stabilizer," Construction and Building Materials, vol. 174, pp. 520-528, 2018.

[12] N. Pervaia, "Structural and performance characteristics of pigskin leather with different tanning methods," Journal of the
Society of Leather Technologists and Chemists, vol. 103, no. 3, pp. 122-128, 2019.

[13] S. Saha and J. Kumar, "Role of interface roughness scattering, temperature, and structural parameters on the performance characteristics of III-nitride quantum cascade detectors," Journal of Applied Physics, vol. 121, no. 5, pp. 053104-153101, 2017.

[14] G. Yang, C. Wang, P. Wen, and W. Yin, "Performance characteristics of cold-mixed porous friction course with composite-modified emulsified asphalt," Journal of Materials in Civil Engineering, vol. 32, no. 3, pp. 1-12, Article ID 04019372, 2020.

[15] A. M. Elsaid, "Experimental study on the heat transfer performance and friction factor characteristics of $\mathrm{Co}_{3} \mathrm{O}_{4}$ and $\mathrm{Al}_{2} \mathrm{O}_{3}$ based $\mathrm{H}_{2} \mathrm{O} /\left(\mathrm{CH}_{2} \mathrm{OH}\right)_{2}$ nanofluids in a vehicle engine radiator," International Communications in Heat and Mass Transfer, vol. 108, pp. 1-18, Article ID 104263, 2019.

[16] V. K. Kanth and S. Raghavan, "A novel Faraday-cage inspired FSS shield for stable resonance performance characteristics," International Journal of Electronics Letters, vol. 8, no. 1, pp. 60-69, 2020.

[17] A. Kausar, Z. Anwar, and B. Muhammad, "Overview of nonflammability characteristics of graphene and graphene oxide-based polymeric composite and essential flame retardancy techniques," Polymer-Plastics Technology and Engineering, vol. 56, no. 5, pp. 488-505, 2016.

[18] P. Wang, T. Yao, Z. Li et al., "A superhydrophobic/electrothermal synergistically anti-icing strategy based on graphene composite," Composites Science and Technology, vol. 198, Article ID 108307, 2020.

[19] L. Zhang, L. Chen, J. Liu, X. Fang, and Z. Zhang, "Effect of morphology of carbon nanomaterials on thermo-physical characteristics, optical properties and photo-thermal conversion performance of nanofluids," Renewable Energy, vol. 99, pp. 888-897, 2016.

[20] H. Xuemei, S. Yukun, and B. Bo, "Fabrication of cubic p-n heterojunction-like $\mathrm{NiO} / \mathrm{In}_{2} \mathrm{O}_{3}$ composite microparticles and their enhanced gas sensing characteristics," Journal of Nanomaterials, vol. 2016, Article ID 7589028, 9 pages, 2016.

[21] K. Hayat, F. Niaz, S. Ali et al., "Thermoelectric performance and humidity sensing characteristics of $\mathrm{La}_{2} \mathrm{CuO}_{4}$ nanofibers," Sensors and Actuators B: Chemical, vol. 231, pp. 102-109, 2016.

[22] X. Yin, H. Li, R. Yuan, L. Zhang, and J. Lu, "General formation of Prussian blue analogue microtubes for high-performance Na-ion hybrid supercapacitors," Science China Materials, vol. 63, no. 5, pp. 739-747, 2020.

[23] S. Choi, S. I. Han, D. Kim, T. Hyeon, and D. H. Kim, "Highperformance stretchable conductive nanocomposites: materials, processes, and device applications," Chemical Society Reviews, vol. 48, no. 15, pp. 1566-1595, 2019.

[24] S. Shariati, M. Abedi, A. Saedi et al., "Critical factors of the application of nanotechnology in construction industry by using anp technique under fuzzy intuitionistic environment," Journal of Civil Engineering and Management, vol. 23, no. 7, pp. 914-925, 2017.

[25] P. Chindaprasirt, "Nanotechnology in eco-efficient construction: materials, processes and applications, 2nd edition," Journal of Zhejiang University-Science A (Applied Physics \& Engineering), vol. 20, no. 5, pp. 382-383, 2019.

[26] V. Komar, T. A. Pochodina, N. V. Kulinich, N. P. Krutko, and L. V. Ovseenko, "Modification of water-suspension epoxy varnish and paint materials by nanostructured magnesium 
oxide," Proceedings of the National Academy of Sciences of Belarus, Chemical Series, vol. 56, no. 1, pp. 105-113, 2020.

[27] G. Alistair, J. Wendy, G. Chris, P. Bust, M. Song, and J. Jin, "Nanotechnology in construction and demolition: what we know, what we don't," Construction Research \& Innovation, vol. 9, no. 2, pp. 55-58, 2018.

[28] V. Vishwakarma and D. Ramachandran, "Green concrete mix using solid waste and nanoparticles as alternatives-a review," Construction and Building Materials, vol. 162, no. 20, pp. 96-103, 2018.

[29] L. Czarnecki, "Is nanotechnology an efficient tool in ecoconstruction?" Journal of Zhejiang University Science A, vol. 20, no. 5, pp. 380-381, 2019.

[30] A. K. Chatterjee, "Nanoparticulate matters in cement, concrete and construction performance and EHS perspectives," Indian Concrete Journal, vol. 91, no. 2, pp. 12-24, 2017.

[31] A. S. Luyt, B. Škipina, L. Csóka, and D. Dudić, "Charge-trapping capability and AC conductivity at different humidities of poly(ethyleneimine)- $\mathrm{TiO}_{2}$-anthocyanin-modified cellulose fibres," Wood Science and Technology, vol. 52, no. 3, pp. 637-651, 2018.

[32] S. Palmieri, G. Cipolletta, C. Pastore et al., "Pilot scale cellulose recovery from sewage sludge and reuse in building and construction material," Waste Management, vol. 100, pp. 208-218, 2019.

[33] W. J. Long, J. L. Tao, C. Lin et al., "Rheology and buildability of sustainable cement-based composites containing microcrystalline cellulose for 3D-printing," Journal of Cleaner Production, vol. 239, pp. 1-13, Article ID 118054, 2019.

[34] J. D. Benck, T. R. Hellstern, J. Kibsgaard, P. Chakthranont, and T. F. Jaramillo, "Catalyzing the hydrogen evolution reaction (HER) with molybdenum sulfide nanomaterials," ACS Catalysis, vol. 4, no. 11, pp. 3957-3971, 2016.

[35] Y. Shi and B. Zhang, "Correction: recent advances in transition metal phosphide nanomaterials: synthesis and applications in hydrogen evolution reaction," Chemical Society Reviews, vol. 45, no. 6, p. 1781, 2016. 